\title{
Sterols from Polyene-resistant Mutants of Candida albicans
}

\author{
By J. M. T. HAMILTON-MILLER* \\ Mycology Unit, Charing Cross Hospital Medical School, London W. 6 \\ (Received 13 March 1972) \\ INTRODUCTION
}

Polyene antibiotics act by combining with sterols in the membrane of susceptible organisms, and thereby affect permeability. Recent studies have indicated that polyene resistance may be associated with altered sterol patterns in Candida spp. (Athar \& Winner, I97I), quantitatively, and in Saccharomyces cerevisiae (Thompson, Starr \& Parks, I97I; Woods, 197I), quantitatively and qualitatively. Following the recent isolation (Hamilton-Miller, 1972) of a new type of polyene-resistant Candida albicans mutant (mutagen-induced), the sterol contents of such mutants were compared with that of the parent strain.

\section{METHODS}

A clinically isolated Candida albicans strain (799-s) was treated with $N$-methyl- $N^{\prime}$-nitro$N$-nitrosoguanidine, and four polyene-resistant mutants, 799-XL, -XS, -YL and -YS, were isolated (Hamilton-Miller, I972). Organisms were grown on Sabouraud agar contained in I $4 \mathrm{~cm}$ Petri plates (two plates for each strain), inoculated with $2 \mathrm{ml}$ amounts of Sabouraud broth overnight cultures and incubated at $37{ }^{\circ} \mathrm{C}$ for $48 \mathrm{~h}$. Organisms were washed off the plates, washed with water and then homogenized in an equal volume of water (2 to $3.5 \mathrm{ml}$ ); I $\mathrm{ml}$ amounts of the resulting suspensions were removed for dry weight determinations, and $2 \mathrm{ml}$ amounts were saponified in ethanolic $\mathrm{KOH}$ and analysed for sterols, as described by Breivik \& Owades (1957). This procedure was followed three times with each of the five strains. Spectra were recorded by using a Unicam SP 800 spectrophotometer.

\section{RESULTS}

As shown in the Table $\mathrm{I}$, strain XL contained significantly more ergosterol than the parent $(P<0.05)$, while differences between the other three strains and the parent were not significant, as far as ergosterol content/mg dry wt is concerned.

Qualitatively, the absorption spectra of hexane extracts of all five strains were virtually identical in the region 300 to $265 \mathrm{~nm}$, and showed the characteristic three-peaked spectrum of ergosterol $\left(\lambda \lambda_{\max }\right.$ at 293, 282 and $\left.271 \mathrm{~nm}\right)$. The extracts from the parent strain and from strains XL and XS showed no other peaks, but those from strains YL and Ys had considerable absorption in the region 255 to $230 \mathrm{~nm}$, with a plateau between 240 and $230 \mathrm{~nm}$ having $E=\mathrm{I} \cdot 5 \times E 282 \mathrm{~nm}$. The spectrum of these two extracts thus closely resembles that of the nys-I a mutant of Saccharomyces cerevisiae described by Woods (I97I).

* Present address: Department of Medical Microbiology, Royal Free Hospital, London, WCi X 8LF. 


\section{Table I. Ergosterol content of parent $(S)$ and four mutants of Candida albicans 799}

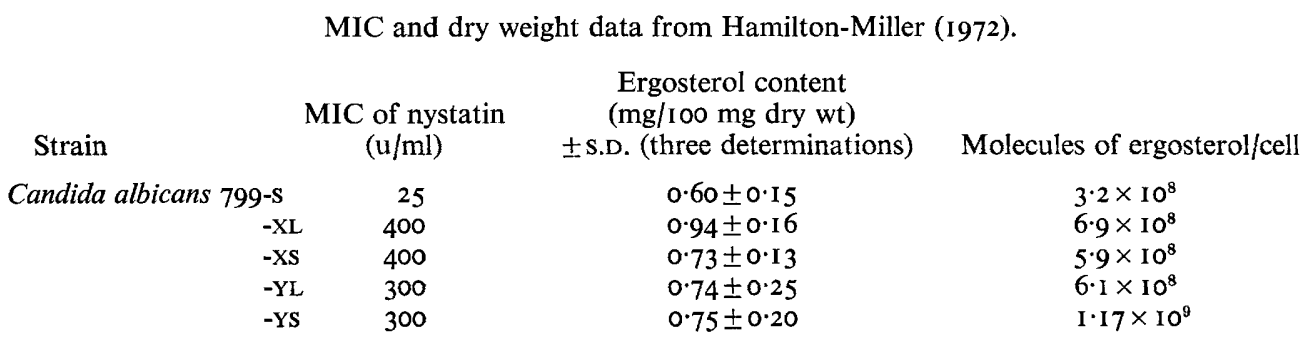

\section{DISCUSSION}

The spectral pattern observed in extracts of strains YL and Ys is similar to, but not identical with, that of 24(28)-dehydroergosterol (which has a sharp maximum at $230 \mathrm{~nm}$, $E_{23} / E_{282}=1.68$; Breivik \& Owades, I957), and is completely different from that of zymosterol (Thompson et al. I97I). Cholesterol, $\beta$-sitosterol and stigmasterol were found to have no absorption maxima in the region 255 to $230 \mathrm{~nm}$, and although lanosterol showed a plateau between 240 and $230 \mathrm{~nm}$ it was of low intensity $\left(E_{\mathrm{I}} \%=29\right)$, and to account for the observed extinction the lanosterol: ergosterol ratio in the extracts of strains YL and YS would have to be about 150: I. It therefore seems probable that a sterol (or sterols) other than those mentioned above is present in strains YL and YS, in addition to ergosterol.

Individual organisms of the mutant strains each possess about 2 to 3 times as much ergosterol as do those of the parent (see Table I); thus, these mutants are unlike both the nys-3 mutants of Saccharomyces cerevisiae (Woods, 197I) and the 'trained' Candida strains (Athar \& Winner, 1971) which appear to be deficient in ergosterol. Nys-I mutants of $S$. cerevisiae (which are more resistant than nys-3 mutants; Woods \& Ahmed, 1968) possess a new sterol (Woods, 197I) in addition to ergosterol, and the Candida albicans mutant strains YL and Ys seem to be analogous to these. Strains XL and Xs may possess an additional sterol with no significant ultraviolet absorption spectrum, and thus be analogous to nys-I mutants, or they may represent a distinct form of polyene resistance which is independent of altered sterol patterns. The answer must await a more detailed analysis of the sterol extracts from the different strains.

I am grateful to Miss P. Thorne for assistance, and to Dr J. Swale for a helpful discussion. This work was carried out during the tenure of a Squibb Research Fellowship.

\section{REFERENCES}

Athar, M. A. \& Winner, H. I. (I971). The development of resistance by Candida species to polyene antibiotics in vitro. Journal of Medical Microbiology 4, 505-5I 7 .

BREIVIK, O. N. \& OWADES, J. L. (1957). Spectrophotometric semimicrodetermination of ergosterol in yeast. Agricultural and Food Chemistry 5, 360-363.

Hamilton-Miller, J. M. T. (1972). Physiological properties of mutagen-induced variants of Candida albicans resistant to polyene antibiotics. Journal of Medical Microbiology (in the press). 
Thompson, E. D., Starr, P. R. \& Parks, L. W. (197I). Sterol accumulation in a mutant of Saccharomyces cerevisiae defective in ergosterol production. Biochemical and Biophysical Research Communications 43, I 304-I 309.

Woods, R. A. (197I). Nystatin-resistant mutants of yeast: alterations in sterol content. Journal of Bacteriology I08, 69-73.

Woods, R. A. \& AHmed, K. A. (1968). Genetically controlled cross-resistance to polyene antibiotics in Saccharomyces cerevisiae. Nature, London 218, 369-370. 